erial can create the strong effects seen in the spectra of these disks. At $2 \mathrm{~mm}$ wavelength, some of the disks emit 30 times the radiation expected from normal interstellar dust particles. Radio radiation is strengthened far more effectively by changing the size and shape of the antenna than by changing its chemical makeup.

Whatever is responsible for the enhanced radiation, there is no doubt that the solid-state constituents in these disks are markedly different from the interstellar dust whence they came. Whether the result of growth or chemical processing, there is strong evidence that the solids are being transformed as they orbit the stars. Such transformations are precisely what we need to see to determine whether the particles make their way into planets. Even if the present work needs more support to define unambiguously the onset of planet formation, we now have a sample of stars for testing our theories, and testable criteria by which we can explore the processes that shaped our own rocky beginning from a dusty past.

Steven V. W. Beckwith is at the Max-PlanckInstitut für Astronomie, Königstuhl 17, 69117 Heidelberg, Germany.

1. Mannings, V. \& Emerson. J. P. Mon. Not. R. astr. Soc. 267 , 361-378 (1994)

. Miyake, K. \& Nakagawa, Y. Icarus (in the press)

3. Aumann, H. H. et al. Astrophys. J. 278, L23-27 (1984).

. Strom. K. M. et al. Astr. J. 97. 1451-1470 (1989)

5. Lynden-Bell. D. \& Pringle, J. E. Mon. Not. R. astr. Soc. 168. 603-637 (1974).

6. Beckwith. S. V. W. \& Sargent, A. I. Astrophys. J. 381, 250 (1991)

Wright, E. L. Astrophys. J. 320, 818-824 (1987)

8. Meakin. P. \& Donn, B. Astrophys. J. 329, L39-42 (1988)

9. Brownlee. D. E. et al. Proc. 8th Lunar Sci. Conf., 149

(Pergamon, New York. 1977).

\title{
Flummoxed by fake fruits
}

\section{Peter D. Moore}

MANY plants depend on animals for the dispersal of their fruits and seeds, and such species usually advertise their wares by the use of bright colours and conspicuous forms. But for others the levels of seed predation can become unacceptably high and selective pressures operate in the reverse direction, forcing the plant to hide or disguise its fruit. One of the most unusual methods of achieving this end is described by P. K. Groom, B. B. Lamont and H. C. Duff in the latest issue of Functional Ecology $(8,110-117 ; 1994)$ - they have identified a plant in which specialized leaves that resemble fruits serve to confuse the birds that seek to strip the plant of its produce.

Plant leaves are remarkably adaptable. Besides their obvious photosynthetic role, they may be modified to protect buds, form spines that deter grazers, construct traps that catch insects, make tendrils that enable a stem to climb, or house secretory organs that attract ants. It has even been argued that variegated leaves in patchy shade operate as camouflage, reducing the conspicuousness of the plant in the eyes of potential grazers, and the same may be true of highly dissected leaves and those with holes in their laminas, such the Swiss cheese plant, Monstera deliciosa. But in the Australian shrub Hakea trifurcata (Proteaceae) the mature shoots develop distinctive leaves with a form that bears a strong resemblance to the follicles that contain the seeds, and Groom, Lamont and Duff propose that these leaves serve to distract and confuse the main predator of the follicles, the white-tailed black cockatoo.

Hakea trifurcata is unusual among the Australian Proteaceae in that its leaves

are dimorphic. The juvenile plant produces only narrow, needle-like leaves but, on maturity, a second type of leaf develops alongside the needle leaves on the same branches. These are broader, rather

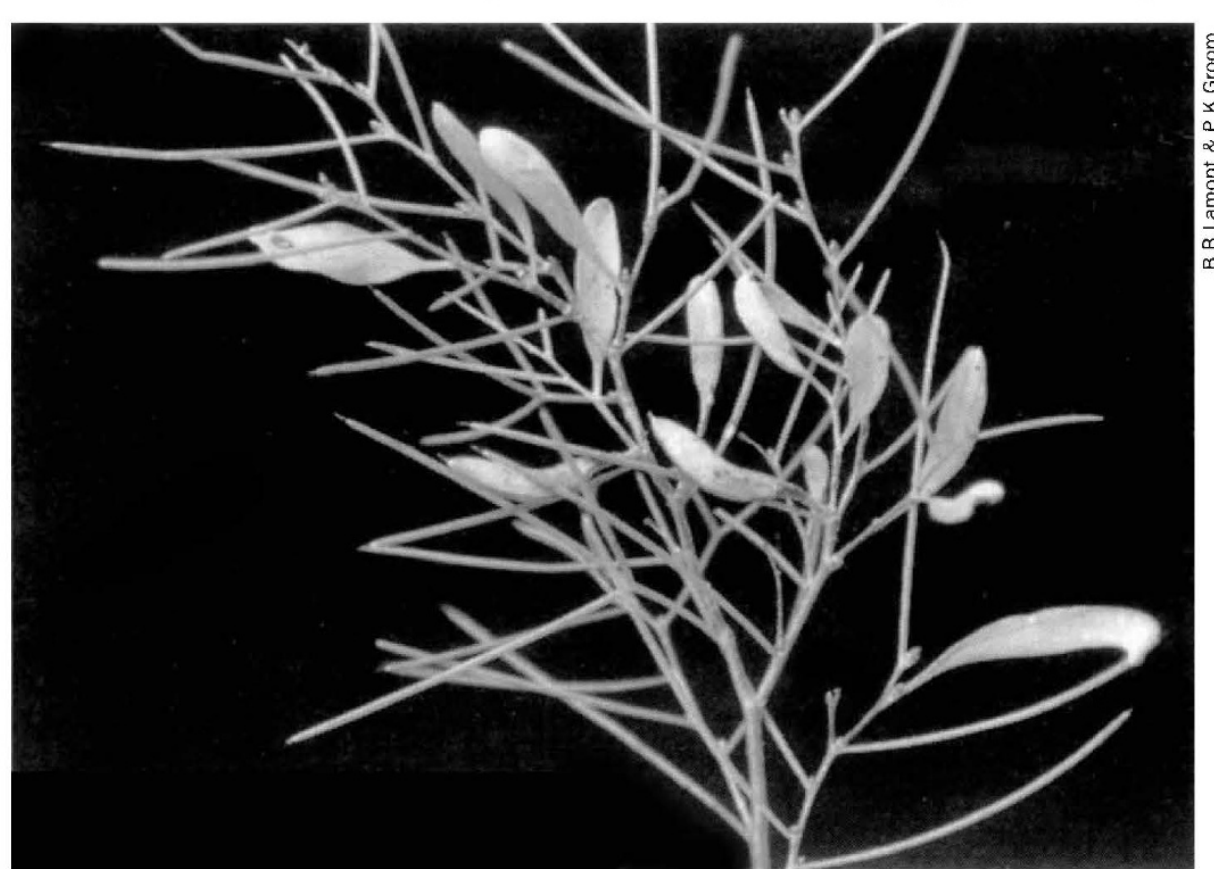

Spot the fruit - this branch of Hakea trifurcata bears two fruits, the rest of the structures being leaves. But which is which? The photograph is about two-thirds life size.

spatulate structures, which in shape and colour (yellow-green) are remarkably like the plant's follicles. The broad leaves are generally somewhat longer than the follicles, but those that are situated closer to the fruits are closer to them in size. They are also denser in those areas where the follicles are present. Protection of the fruits from excessive predation makes sense in this species because, unlike most members of the Proteaceae, it is unable to resprout after fires and is entirely dependent on seed production for population recovery.

To test the hypothesis that the broad leaves make it more difficult for cockatoos to locate and harvest Hakea fruits, caged birds were subjected to feeding trials in which they were offered normal fruiting branches with broad leaves present, and treated fruiting branches with all broad leaves removed. The total number of follicles subsequently removed by the birds from treated branches was significantly higher than in those with broad leaves present. Overall, the birds approached the broad leaves in the untreated branches two to three times more often than real fruits, perhaps being further distracted by the generally larger size of the deceptive leaves, especially those at greater distances from the genuine article. Having developed a search image appropriate for the detection of Hakea follicles, the birds are evidently easily led astray by the cryptic leaves.

Mimicry is, of course, quite common in nature, though self-mimicry, or 'selfcrypsis' as the authors term it, is most unusual, if not unique, among plants. The evolution of this strategy in Hakea trifur- cata demonstrates that the energy invested in deception evidently pays off, and the moral of the story seems to be that if you really want to confuse a predator, then go around looking like yourself.

Peter D. Moore is in the Division of Life Sciences, King's College, Campden Hill Road, London W8 7AH, UK. 